\title{
Acute functional reorganisation of the human motor cortex during resection of central lesions: a study using intraoperative brain mapping
}

\author{
H Duffau
}

\begin{abstract}
Objectives-Brain plasticity is supposed to allow the compensation of motor function in cases of rolandic lesion. The aim was to analyse the mechanisms of functional reorganisation during surgery in the central area.

Methods-A motor brain mapping was performed in three right handed patients without any neurological deficit, operated on for a slow growing lesion near the rolandic region (two precentral resected under general anaesthesia and one retrocentral removed under local anaesthesia to allow also sensory mapping) using intraoperative direct electrical stimulations ( $5 \mathrm{~mm}$ space tips bipolar stimulator probe, biphasic square wave pulse current: $1 \mathrm{~ms} / \mathrm{phase}, 60 \mathrm{~Hz}, 4$ to $18 \mathrm{~mA}$ ).

Results-For each patient, the motor areas of the hand and forearm in the primary motor cortex (M1) were identified before and after lesion removal with the same stimulation parameters: the same eloquent sites were found, plus the appearance after resection of additional sites in M1 inducing the same movement during stimulations as the previous areas. Conclusions-Multiple cortical representations for hand and forearm movements in M1 seem to exist. In addition, the results demonstrate the short term capacity of the brain to make changes in local motor maps, by sudden unmasking after tumour resection of a second redundant site participating in the same movement. Finally, it seems not necessary for the whole of the redundant sites to be functional to provide normal movement, a concept with potential implications for surgery within the central region.

(F Neurol Neurosurg Psychiatry 2001;70:506-513)
\end{abstract}

Service de

Neurochirurgie 1,

Hôpital de la

Salpêtrière, $47 \mathrm{Bd}$ de

l'hôpital, 75651 Paris,

Cedex 13, France

H Duffau

Correspondence to:

Dr H Duffau

hugues.duffau@

psl.ap-hop-paris.fr

Received 1 June 2000 and in revised form

23 October 2000

Accepted 2 November 2000 lesions. This recruitment of adjacent corticospinal motor output neurons was explained by a recent concept of a dynamic pattern of $\mathrm{M} 1$, with multiple and overlapping representations for hand muscles and movements. ${ }^{12}{ }^{13}$ From physiological data reviewed previously, ${ }^{14}$ it seems that a horizontal connectivity may exist among local circuits linking pyramidal cells (between layers III or V), leading to a simple model of latent intracortical connections that could provide a substrate for remodelling intrinsic circuitry. Indeed, this model for plasticity predicts that reduced inhibition (particularly in M1 lesions) would tend to strengthen the coupling of reciprocally connected pyramidal cells, with unmasking of a new motor output architecture. ${ }^{15}$

However, this mosaic-like representation was only illustrated experimentally ${ }^{15-17}$ or using methods of functional neuroimaging in normal subjects. ${ }^{12}$ Thus, the hypothesis of within motor system recovery by new relations between local circuits and muscle grouping ${ }^{14}$ in the case of an M1 lesion-labelled the "plasticity of the motor cortex"- ${ }^{6}$ was only indirectly assumed in humans, by studies using functional neuroimaging methods comparing the displacement of cortical hand and arm representations with regard to the expected normal location $^{18}$ (although reorganisation of the

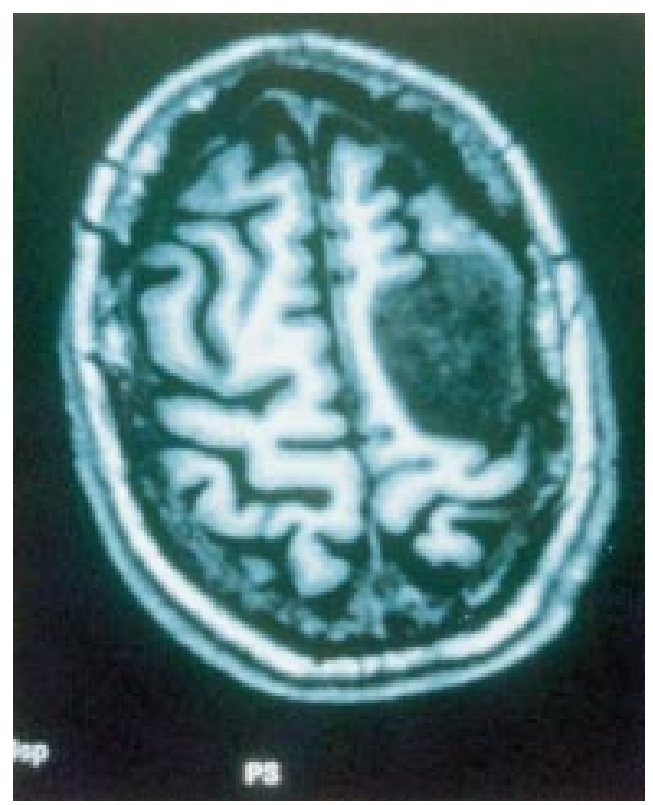

Figure 1 Preoperative axial T1 weighted enhanced MRI in a 42 year old woman with a normal neurological examination, showing a left precentral low grade glioma. 


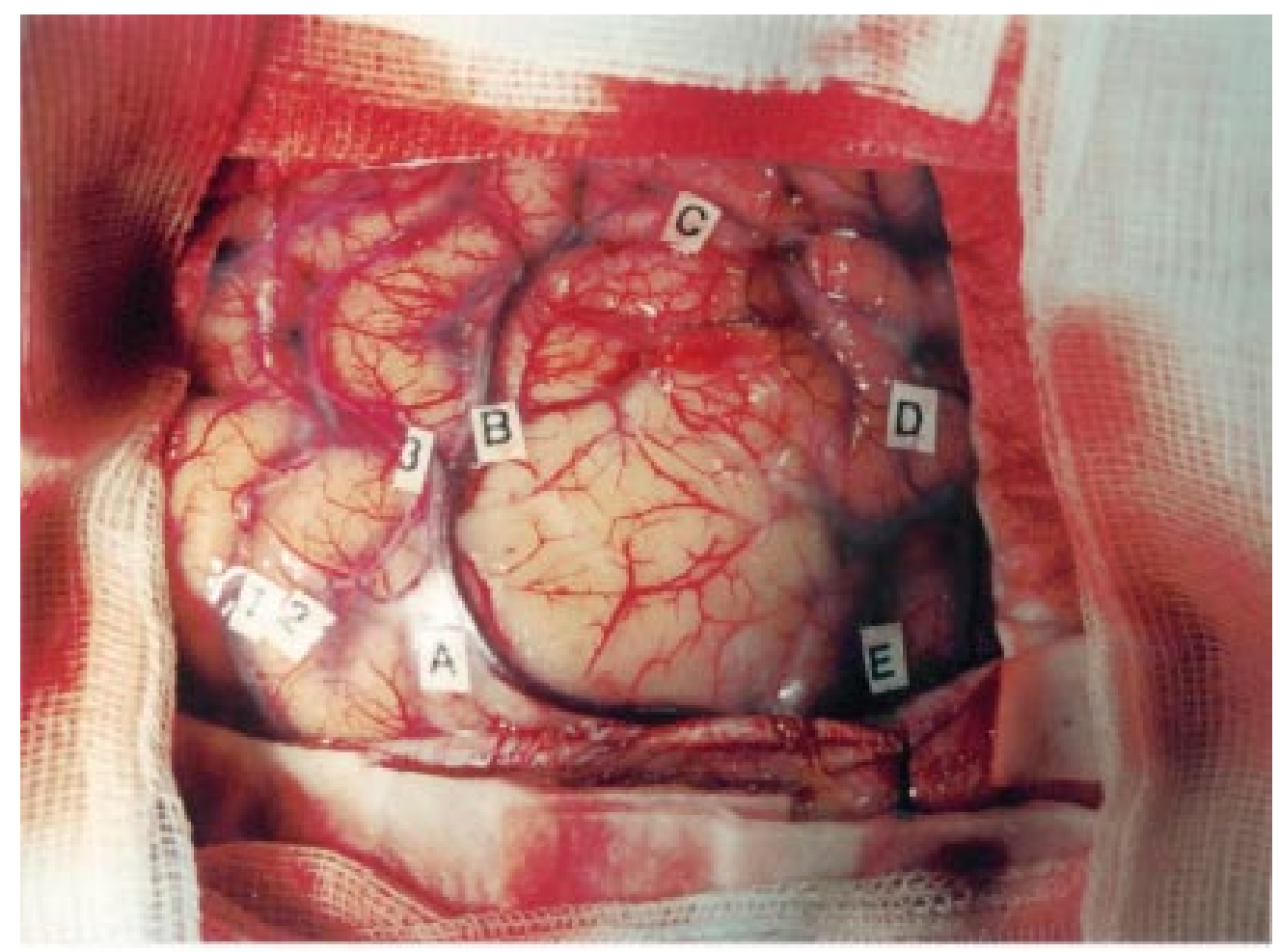

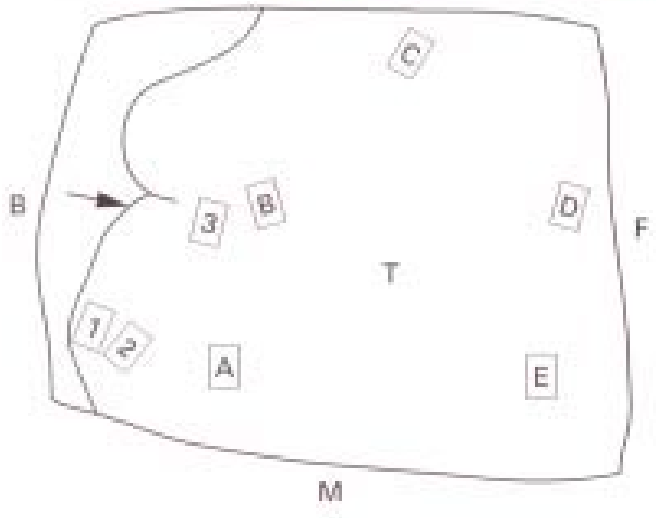

motor map has been clearly demonstrated after M1 lesion in monkeys). ${ }^{19}$

From an intraoperative study of cortical motor mapping using cerebral electrical stimulations before and after surgical resection of a lesion near the central region in three patients, I describe direct evidence for a short term local unmasking of additional motor areas. On the basis of these findings, I discuss the potential implication of this phenomenon in the compensatory plasticity mechanisms in humans, in lesions in functional regions and their surgical treatment.

\section{Materials and methods}

Three right handed women $(32,42$, and 55 years of age) underwent surgery in our institution for a brain lesion discovered after seizures. All lesions were slowly progressive; two were low grade gliomas and one an arteriovenous malformation. Neurological examination was normal in all patients, without any motor disturbance. One glioma had a precentral location; the vascular malformation had a rolandic site; and the other glioma was postcentral.
Figure 2 Intraoperative preresection view photography (top) and drawing (bottom) showing three functional sites in M1 behind the lesion, inducing during stimulation $(18 \mathrm{~mA})$ a controlateral motor response of respectively the forearm (1), the wrist (2), and the fingers (3) marked with numbered tags. No other response was obtained. The shoulder and hip areas were likely more medially located and then unexposed by the bone flap. The face areas are known to be often difficult to identify under general anaesthesia, due to the intubation masking the face movements. Letters represent the limits of the tumour, detected using ultrasonography. $A=$ Angioma; $B=$ backward; $C=$ cavity; $F=$ forward; $M=$ midline; $T=$ tumour; arrow =central sulcus.

Surgery was performed under general anaesthesia without curarisation, with motor mapping in the two patients with precentral and central lesions (anaesthetic levels constant during all the procedure), and under local anaesthesia on an awake patient (with the retrocentral tumour) with the possibility of motor and somatosensory mapping (no anaesthetic agent during all the resection). In all patients, the lesion was first localised after opening the dura mater, by eye for the readily apparent vascular malformation, and with the help of ultrasonography for the two gliomas (the boundaries were marked with sterile letter tags).

Before any resection, a motor cortical mapping using direct electrical stimulations was carried out, previously described in the literature as a safe, reliable, and accurate (without any diffusion) method. ${ }^{20-24}$ This was done using a $5 \mathrm{~mm}$ spaced tips bipolar stimulator probe (Ojemann Cortical Stimulator, Radionics ${ }^{\star}$. Stimulation parameters were set at $60 \mathrm{~Hz}$, biphasic square wave pulses ( $1 \mathrm{~ms} /$ phase), with 


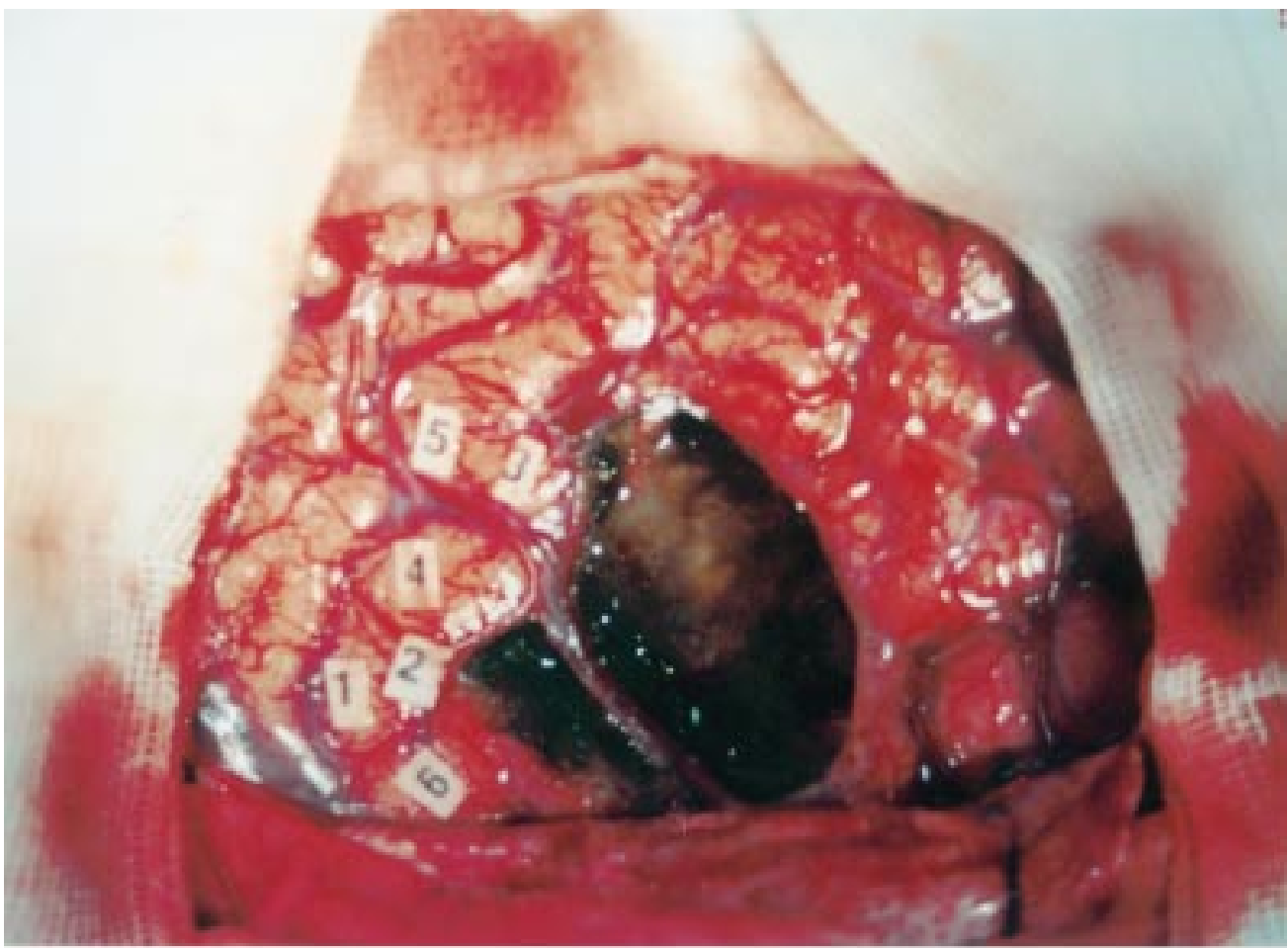

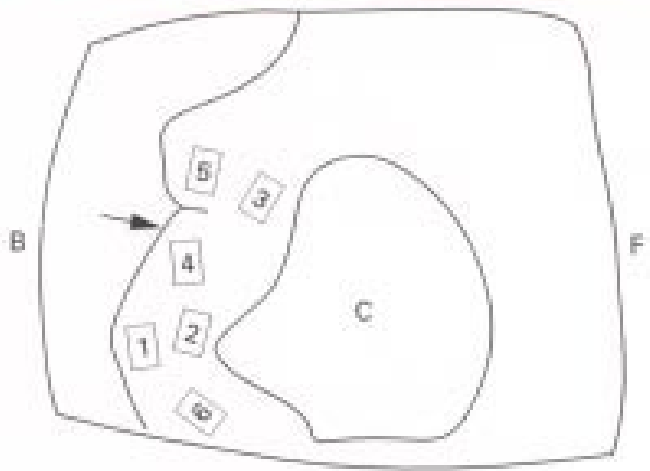

M

a progressive increase in the current amplitude (from $2 \mathrm{~mA}$ ) until a motor (or sensory in one patient, see below) response was obtained. An intensity of $18 \mathrm{~mA}$ was necessary in the two patients under general anaesthesia $(18 \mathrm{~mA}$ being the highest level considered as nondeteleterious for the brain), and an intensity of $4 \mathrm{~mA}$ in the awake patient. The duration of

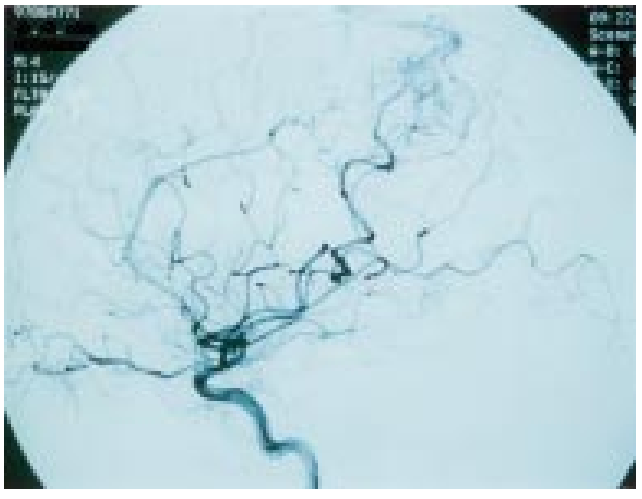

Figure 4 Preoperative angiography showing a left retrocentral arteriovenous malformation.
Figure 3 Intraoperative postresection view photography (top) and drawing (bottom) shows that the same responses as before resection were found with the same electrical parameters $(18 \mathrm{~mA})$ at the same three sites (1,2, and 3), with tags 2 and 3 slighty raised (respectively from 2 and $3 \mathrm{~mm}$ ) because of the anatomical deformation due to the tumour resection (shift). There were also three new sites inducing the same motor response of the wrist (4), the fingers (5), and the forearm (6), a distance $1 \mathrm{~cm}$ from the previous areas 1,2, and 3. There were no other new responses obtained by stimulation of the remaining primary motor cortex. $A=$ Angioma; $B=$ backward $C=$ cavity;

$F=$ forward $M=$ midline; arrow = central sulcus.

each brain stimulation was 1 second. Motor responses of the fingers, the wrist, and the forearm were clinically obtained in all patients and, in the awake patient, a somatosensorial map was also obtained, by inducing contralateral sensations described by the patient himself. Responsive cortical sites were marked using number tags.

Resection was then performed, sparing the functional areas detected by stimulations, regularly given during the resection, to define precisely the interface between lesion and functional areas. At the end of the resection, cortical stimulations were repeated using the same electrical parameters (particularly, the same intensity) as before, to check the functional integrity of the pyramidal pathways again, with the same clinical criteria of evaluation used previously.

\section{Results}

In the first patient, with a left precentral low grade glioma (fig 1) and normal neurological examination, intraoperative preresection mapping found three functional sites in $\mathrm{M} 1$ (behind 


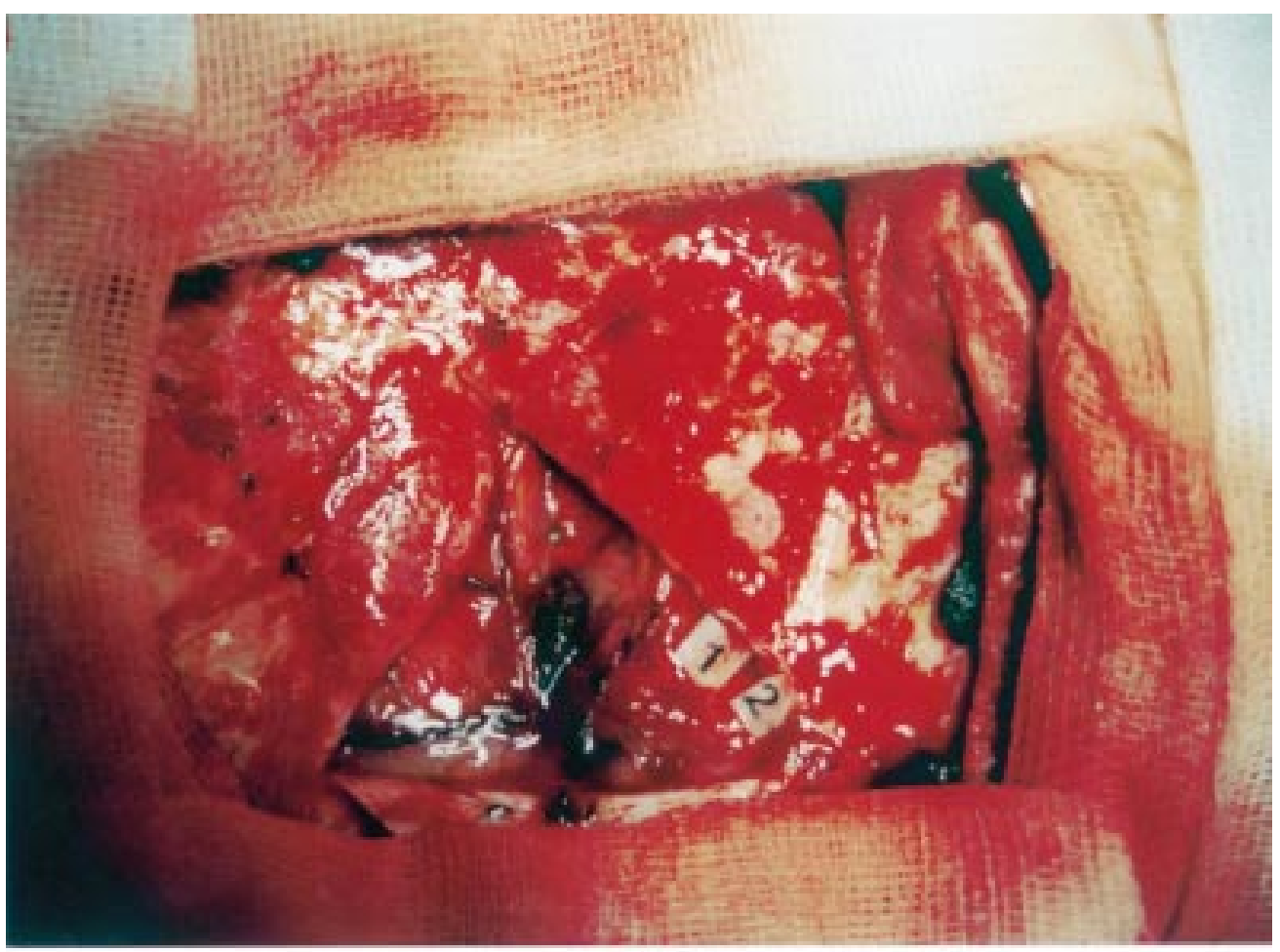

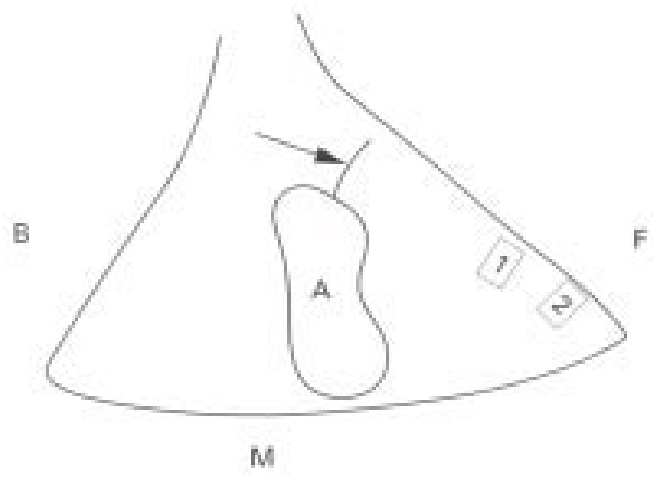

the lesion), inducing motor responses of the forearm, wrist, and fingers (fig 2), without any other response during stimulations of the adjacent exposed areas. Cortical stimulations using the same electrical parameters after tumour removal showed the same motor responses at the same three sites as before, plus three new redundant areas in $\mathrm{M} 1$ also generating exactly the same movements respectively of the forearm, wrist, and fingers (fig 3 ).

In the second patient, without clinical abnormalities, with a left rolandic arteriovenous malformation (fig 4), intraoperative preresection mapping showed two motor sites in front of the lesion, inducing movement of the fingers and wrist (fig 5). Again, postresection stimulations with the same intensity level found the same two motor sites, plus one new redundant functional area in M1 inducing exactly the same motor response of the wrist than the previous site (fig 6).

In the third patient without neurological deficit, harbouring a left postcentral low grade glioma (fig 7), and operated on awake under local anaesthesia with the goal of performing
Figure 5 Intraoperative preresection view photography (top) and drawing (bottom) demonstrating two functional sites in M1 in front of the lesion, inducing during stimulation $(18 \mathrm{~mA}$ ) controlateral motor response of respectively the fingers (1) and wrist (2) again marked with tags with a number. No other response was obtained. The angioma was identified by eye as it was readily apparent. $A=$ Angioma; $B=$ backward; $F=$ forward; $M=$ midline; arrow =central sulcus.

intraoperative motor and somato-sensory mapping (taking account of the location of the lesion), preresection cortical stimulations found four functional sites: two located in $\mathrm{M} 1$, inducing motor response of the wrist and fingers, and two postcentral inducing sensitive responses in the hand and the fingers (fig 8). Again, postresection mapping showed the same four eloquent areas, plus two new redundant motor sites in M1 generating exactly the same movement of the wrist and the fingers as the two previous motor areas (fig 9).

The type of the movement (flexion in all cases) and the amplitude (same before and after resection in the three patients) were quoted by an observer, but without objective quantification by electromyography.

The median distance between each "pair" of redundant (new and previous) sites was $1 \mathrm{~cm}$ (range 0.8 to $1.2 \mathrm{~cm}$ ). The median time between the first mapping and the unmasking of new motor sites was about 30 minutes.

No partial or generalised seizure and no oedema was induced during the procedure. 

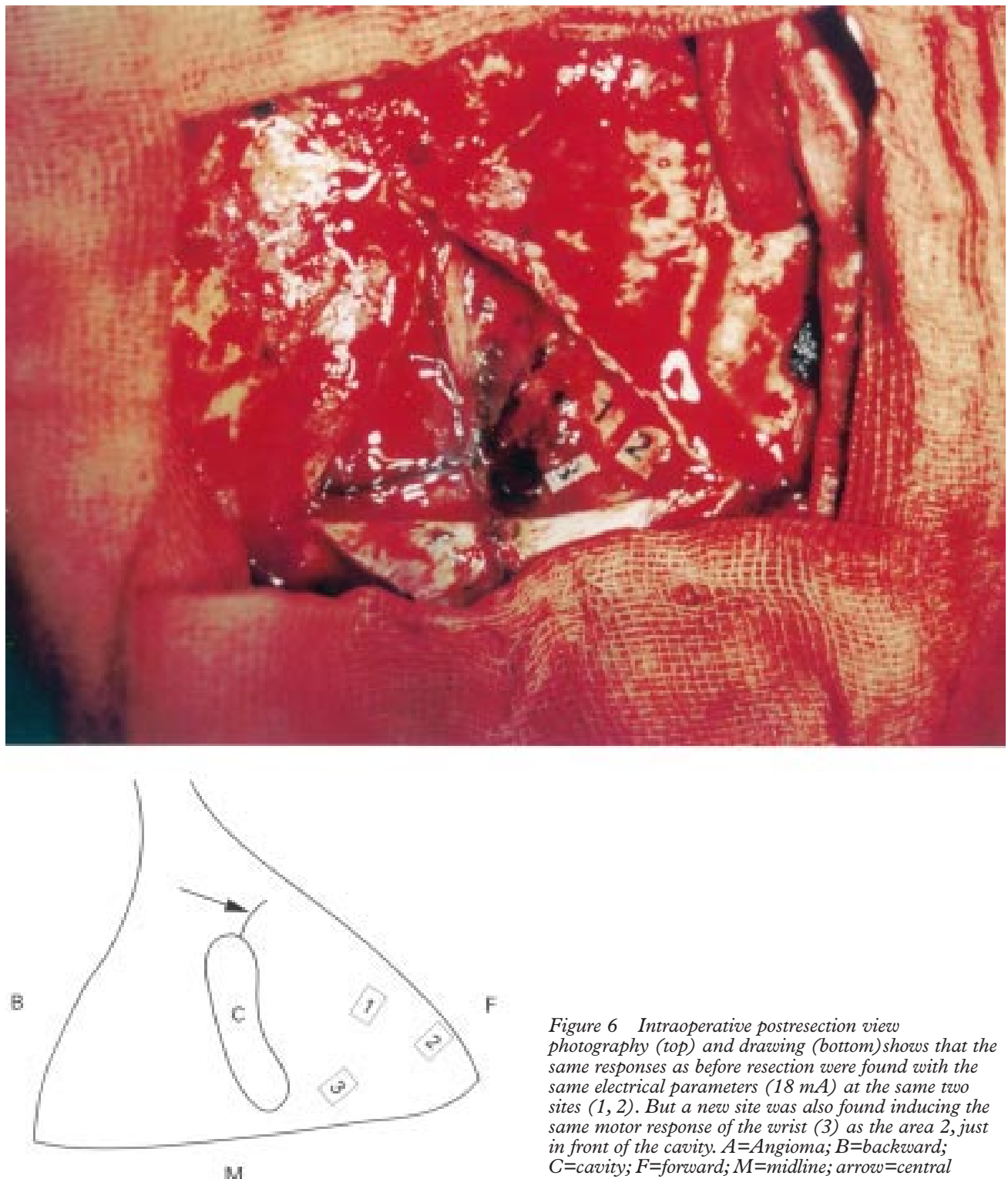

Figure 6 Intraoperative postresection view photography (top) and drawing (bottom) shows that the same responses as before resection were found with the same electrical parameters $(18 \mathrm{~mA})$ at the same two sites $(1,2)$. But a new site was also found inducing the same motor response of the wrist (3) as the area 2, just in front of the cavity. $A=$ Angioma; $B=b a c k w a r d ;$ $C=$ cavity; $F=$ forward; $M=$ midline; arrow $=$ central sulcus.

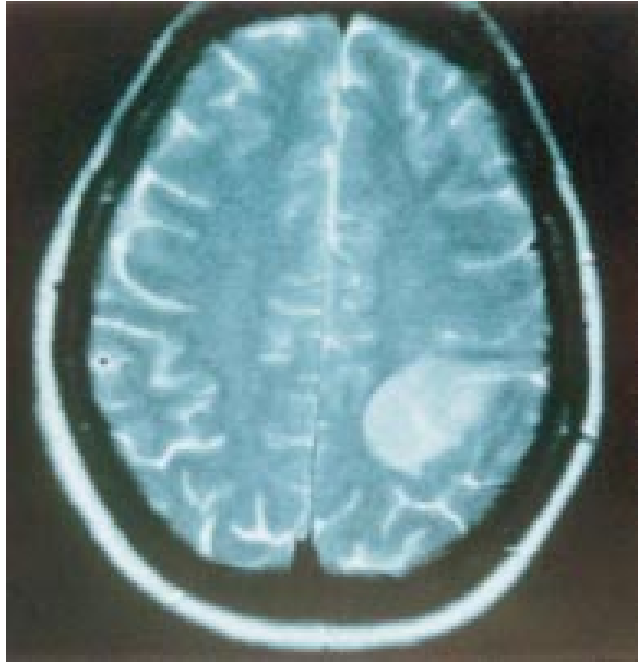

Figure 7 Preoperative axial T2 weighted enhanced MRI in a 55 year old woman with a normal neurological examination, showing a left retrocentral low grade glioma.

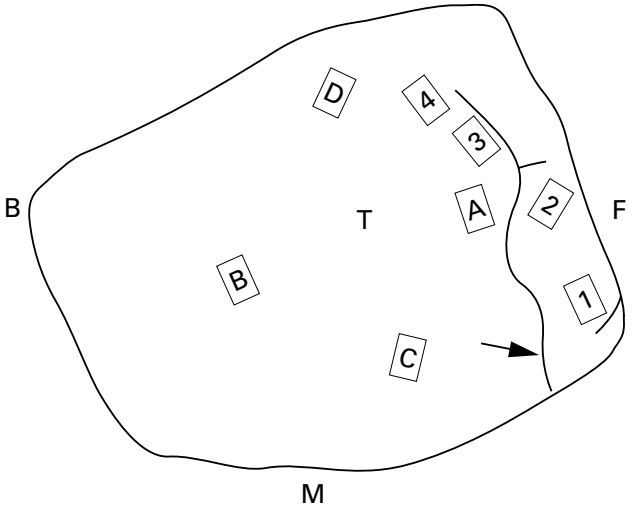

Figure 8 Intraoperative preresection drawing demonstrating four functional sites detected by direct stimulations ( $4 \mathrm{~mA}$ ) under local anaesthesia on the awake patient: two located in M1, inducing a controlateral motor response of respectively the wrist (1) and the fingers (2), and two retrocentral areas (in front of the tumour) eliciting sensitive responses in the hand (3), and the fingers (4). No other response was obtained. Letters identify the limits of the tumour, detected using ultrasonography. A=Angioma; $B=$ backward; $C=$ cavity; $F=$ forward; $M=$ midline; $T=$ tumour; arrow $=$ central sulcus. 


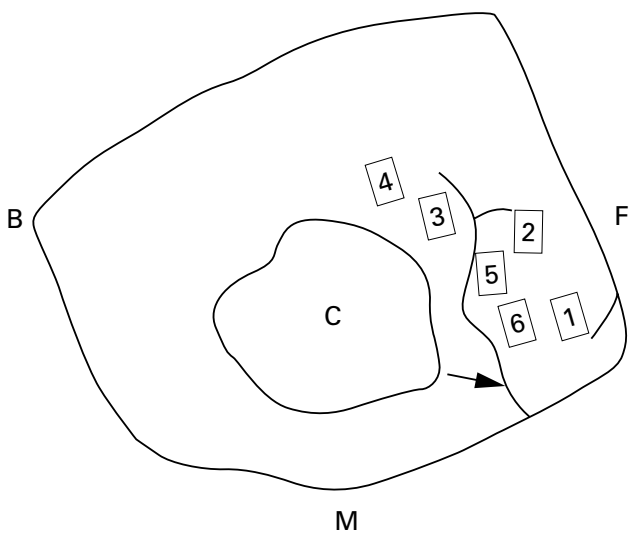

Figure 9 Intraoperative postresection drawing shows that the same responses as before resection were found with the same electrical parameters ( $4 \mathrm{~mA}$ ) at the same four sites

$(1,2,3$, and 4), again with anatomical deformation from forward to backward and from the left to the midline, due to the wide tumour resection. Two new redundant motor sites were also found inducing the same movement of the wrist (6) and the fingers (5) as the previous motor areas 1 and 2. There were no other new responses obtained by stimulation of the remaining primary motor and somatosensory cortex. $B=$ backward; $C=$ cavity; $F=$ forward $M=$ midline; arrow =central sulcus. Intraoperative photographs were not available for technical reasons in the third patient

Postoperative neurological examination was normal in all patients, immediately and 6 months after surgery.

Surgical removal was considered as total in all patients on the postoperative MRI (and on the postoperative angiography in the patient with the angioma).

\section{Discussion}

These findings firstly confirm the existence of multiple cortical representations of finger, wrist, and forearm movements in the primary motor cortex, recently illustrated by functional imaging in normal subjects. Indeed, Sanes et $a l^{12}$ reported using functional MRI, the existence of at least two spatially distinct primary and secondary clusters for each movement in M1, with the possibility of overlapping between movements. This view contrasts with an early somatotopic point by point plan described by Penfield using intraoperative electrical mapping, ${ }^{25}$ holding that representations of each arm segment have a single, contiguous focus of representation. However, it is interesting to note that Penfield had already noted a phenomena of "instability of the motor point", but without precise conclusion. Indeed, this "instability" was detected within a radius of only a few millimeters (and not $1 \mathrm{~cm}$ as in our work), then potentially due to the use of monopolar stimulations which can generate spread of current with the possibility of recruiting networks of adjacent neurons. On the other hand, the method of optical imaging has clearly demonstrated that the bipolar stimulations (used in the present work) do not diffuse, ${ }^{22}$ allowing an accuracy of mapping of 5 $\mathrm{mm}$, thus less than the distance between 2 redundant sites $(1 \mathrm{~cm})$. Moreover, such multiple motor representations have also been reported in primates. ${ }^{26-34}$
These findings also demonstrate the short term capacity of the brain for sudden unmasking of a second functional cluster participating in the same finger, wrist, or forearm movements. Changes in short term mapping have already been reported experimentally. ${ }^{15}$ It was assumed that although long term changes occurring for at least as long as 5 months are likely due to anatomical reorganisation with neosynaptogenesis within $\mathrm{M} 1$, the rapid changes in M1 output occurring within hours likely reflect modifications in synaptic efficacity or switches in local network properties. ${ }^{17}$ This phenomenon of rapid unmasking of eloquent networks, previously advocated as participating to the mechanisms of functional redistribution in lesions of the motor brain area, ${ }^{11}$ was nevertheless only directly illustrated to our knowledge in animals, ${ }^{16}$ or in humans using transcranial magnetic stimulation exclusively in cases of skill acquisition or peripheral deafferentation. ${ }^{135}$ Our results give further arguments in favour of the existence of latent parallel motor redundant circuits, potentially "unmaskable", directly and in real time (intraoperatively) in humans harbouring tumours or malformation of the central region.

On the other hand, it seems important to underline that no electrocorticographic monitoring was performed in this work for assessing potential activity. Accordingly, taking account of the fact that the high settings of current intensity used are close to the levels that are commonly seen as afterdischarges and local seizure activity, the present findings could simply be due to the current spread with depolarisation occurring with afterpotentials due to the increased irritability of the cortex after resection of the tumour. However, the first point is that the intensity of stimulation was progressively increased without two consecutive stimulations at the same site-because of the existence of a well known interindividual variability in the minimal threshold (depending on the patient, the type of lesion, and the anaesthetic condition-local or general anaesthesia)-until a motor or a sensory response was obtained. It is nowadays admitted that there is usually no afterdischarge before the first clinical sensorimotor response-but not for language mapping due to functional inhibition and not activation in this case $\mathrm{e}^{20}{ }^{21}$ consequently without obligation to perform intraoperative electrocorticography to obtain a reliable sensorimotor mapping. ${ }^{20}$ Secondly, there were no partial or generalised seizures generated during the three procedures (easily detectable on the awake patient, and also under general anaesthesia as in this location, even partial seizures should induce movement). Thirdly, the median distance between two redundant sites was $1 \mathrm{~cm}$, thus involving no directly adjacent areas. These data do not suggest that the current spread is the mechanism of the present findings.

The meaning of such a phenomenon of unmasking remains unclear. It could be hypothesised, by the theory of multiple cortical motor sites, that the mass effect of the tumour may have inhibited the second site (or its meta- 
bolic activity) and this redundancy is unmasked after debulking of the tumour. This mechanism of reappearance of cortical function after mechanical decompression of an eloquent motor site was previously described in rapid growing lesions - for example, in metastases. Indeed, we had also operated on, in the same period, six patients with precentral metastases, ${ }^{21}$ for which stimulations gave no response before resection, with the appearance of cortical motor areas detectable by the same stimulations after removal. The difference in these cases is the existence of a motor deficit before surgery with immediate recovery after the operation: we did not find a compensatory "redundant focus" for the same function as the compressed site before resection, which may explain the preexisting paresia, and the clinical recovery after metastasis resection (after reappearance of the corresponding cortical site function, identified by stimulations). There is then no compensatory phenomenon in these cases, probably due to the rapid growth of the metastasis, in opposition to slow growing low grade glioma or congenital arteriovenous malformation.

In the present study, the lack of neurological deficit despite the "masking" of the second site by the tumour before resection, is an argument in favour of the fact that the motor function is likely compensated by the other homologous cortical focus in $\mathrm{M} 1$, then that the functionality of the whole of the redundant sites is not essential to provide normal movement. Nevertheless, it remains unclear whether the additional site unmasked during surgery is:

- One "secondary" accessory eloquent cluster masked by the lesion (according to the concept of a hierarchical organisation of the motor cortex suggested by Sanes et $a l^{12}$ ) without any repercussion on the normal and sufficient functioning of the "primary cluster" (detected by stimulation before resection).

- Or the "primary" functional cluster compensated by the "secondary clusters" recruited during the tumour growth?

If the second hypothesis defines the real situation, it means that the intraoperative unmasking might correspond to the detection of a "reactivation " of the "primary" cluster, and then could illustrate a local brain plasticity phenomenon.

Answers to this question seem crucial for the neurosurgeron. Indeed, if the first hypothesis constitutes the real mechanism, it could then become possible if necessary (for instance in case of tumour infiltration), to surgically remove the "secondary cluster", allowing improvement in the quality of resection in M1 without neurological deficit. One perspective to resolve this problem could be the realisation under local anaesthesia of an alternative transitory inhibition of each redundant site, using a method of cooling already reported as accurate and safe in monkeys, ${ }^{36}$ and to observe the clinical motor consequences on the awake patients.

\section{Conclusions}

These findings give further arguments for the existence of multiple cortical representations for hand and forearm movements in M1. In addition, they demonstrate the short term capacity of the brain for local motor map changes, by sudden unmasking after tumour resection of a second redundant site participating in the same movement. Finally, these data seem to show that it is not necessary for the whole of the redundant sites to be functional to provide normal movement, a concept potentially useful in surgery near or within the motor region.

1 Cohen L, Pascual-Leone A, Hallet M. Plasticity of cortical motor output organization following deafferentation, cerebral lesion, and skill acquisition. In: Devinsky O, Beric A, Dogali M, eds. Advances in neurology : electrical and magnetic stimulation of the brain and the spinal cord. New York: Raven Press, 1993:187-201.

2 Fisher CM. Concerning the mechanisms of recovery in infarct hemiplegia. Can f Neurol Sci 1992;19:57-63.

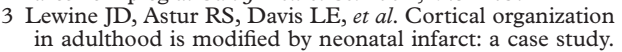
in adulthood is modified by

4 Mogilner A, Grossman JAI, Ribary U, et al. Somatosensory cortical plasticity in adult human revealed by magnetoencephalography. Proc Natl Acad Sci USA 1993;90:3593-7.

5 Ramachandran VS. Behavorial and magnetoencephalographic correlates of plasticity in the adult human brain. Proc Natl Acad Sci USA 1993;90:10413-20.

6 Seitz RJ, Huang Y, Knorr U, et al. Large -scale plasticity of the human motor cortex. Neuroreport 1995;6:742-4.

7 Weiller C, Chollet F, Friston KJ, et al. Functional reorganization of the brain in recovery from striatocapsular infarction in man. Ann Neurol 1992;31:463-72.

8 Benecke R, Meyer BU, Freund HJ. Reorganization of descending motor pathways in patients after hemispherectomy and severe hemispheric lesions demonstrated by magnetic brain stimulation. Exp Brain Res 1991;83:41926 .

9 Lammers T, Netz J, Hömberg V. Disinhibition of ipsilateral MEP-responses in stroke patients. Electroencephalogr Clin MEP-responses in stroke pat
Neurophysiol 1995;97:S193.

10 Turton A, Wroe S, Trepti N, et al. Ipsilateral EMG responses to transcranial magnetic stimulation during recovery of arm and hand function after stoke. Electroencephalogr Clin Neurophysiol 1995;97:S192.

11 Sanes JN, Donoghue JP. Static and dynamic organization of motor cortex. In: Freund HJ, Sabel BA, Witte OW, eds. Brain plasticity, advances in neurology. Vol 73. Philadelphia: Lippincott-Raven, 1997:277-96.

12 Sanes JN, Donoghue JP, Edelman RR, et al. Shared neural substrates controlling hand movements in human motor cortex. Science 1995;268:1775-7.

13 Schieber MH, Hibbard LS. How somatotopic is the motor cortex hand area? Science 1993;261:489-92.

14 Donoghue JP, Hess G, Sanes JN. Motor cortical substrates and mechanisms for learning. In: Bloedel JR, Ebner TJ, Wise SP, eds. Acquisition of motor behavior in vertebrates. Wise SP, eds. Acquisition of motor behavior

15 Jacobs KM, Donoghue JP. Reshaping the cortical motor map by unmasking latent intracortical connections. Science 1991;251:944-7.

16 Donoghue JP, Suner S, Sanes JN. Dynamic organization of primary motor cortex output to target muscles in adult rats. II. Rapid reorganization following motor nerve lesions. Exp Brain Res 1990;79:492-503.

17 Sanes JN, Wang J, Donoghue JP. Immediate and delayed changes of rat motor cortical output representation with new forelimb configurations. Cereb Cortex 1992;2:141-52.

18 Wunderlich G, Knorr U, Herzog H, et al. Precentral glioma location determines the displacement of cortical hand representation. Neurosurgery 1998;42:18-27.

19 Nudo RJ, Milliken GW. Reorganization of movement representations in primary motor cortex following focal ischemic sentations in primary motor cortex following focal ischemic
infarct in adult suirrel monkey. $f$ Neurophysiol 1996;75: infarct in

20 Berger MS, Ojemann GA. Intraoperative brain mapping techniques in neuro-oncology. Stereotact Funct Neurosurg 1992;58:153-61

21 Duffau H, Capelle L, Sichez JP, et al. Intraoperative direct electrical stimulations of the central nervous system: the Salpêtrière experience with 60 patients. Acta Neurochir (Wien) 1999;141:1157-67.

22 Haglund MM, Ojemann GA, Blasdel GG. Optical imaging of bipolar cortical stimulation. F Neurosurg 1993;78:785-

23 Ojemann GA, Ojemann JG, Lettich E, et al. Cortical language localization in left, dominant hemisphere. An electrical stimulation mapping investigation in $117 \mathrm{pa}$ tients. F Neurosurg 1989;71:316-26.

24 Skirboll SS, Ojemann GA, Berger MS, et al. Functional cortex and subcortical white matter located within gliomas. Neurosurgery 1996;38:678-85. 
25 Penfield W, Bolchey E. Somatic motor and sensory representation in the cerebral cortex of man as studied by

26 Andersen P, Hagan PJ, Phillips GG, et al. Mapping by microstimulation of overlapping projections from area 4 to motor units of the baboon's hand. Proc R Soc Lond B Biol Sci $1975 ; \mathbf{1 8 8 : 3 1 - 6 .}$

27 Asanuma H, Rosen I. Topographical organization of cortical efferent zones projecting to distal forelimb muscles in the monkey. Exp Brain Res 1972;14:243-56.

28 Donoghue JP, Leibovic S, Sanes JN. Organization of the forelimb area in squirrel monkey motor cortex: representation of digit, wrist and elbow muscles. Exp Brain Res 1992;89:1-19.

29 Gould HJ, Cusick CG, Pons TP, et al. The relationship of corpus callosum connections to electrical stimulations maps of motor, supplementary motor, and the frontal eye fields in owl monkeys. F Comp Neurol 1986;247:297325.

30 Humphreys DR. Representation of movements and muscle within the primate precentral motor cortex: historical and current perspectives. Federation Proceedings 1986;45:2687-99.
31 Kwan HC, MacKay WA, Murphy JT, et al. Spatial organization of precentral cortex in awake primates. II. Motor out41:1120-31.

32 Phillips CG, Porter R. The pyramidal projection to motoneurones of some muscle groups of the baboon' forelimb. Prog Brain Res 1964;12:222-42.

33 Sato KC, Tanji J. Digit-muscle responses evoked from multiple intracortical foci in monkey precentral motor cortex. $\mathcal{F}$ Neurophysiol 1989;62:959-70.

34 Waters RS, Samulack DD, Dykes RW, et al. Topographic organization of baboon primary motor cortex: face, hand, forelimb, and shoulder representations. Somatosens Mot Res 1990;7:485-514.

35 Brasil-Neto JP, Cohen LG, Pascual-Leone A, et al. Rapid reversible modulation of human motor outputs after transient deafferentation of the forearm: a study with 1302-6.

36 Sasaki K, Gemba H. Compensatory motor function of the somatosensory cortex for the motor cortex temporarily impaired by cooling in the monkey. Exp Brain Res 1984;55: 60-8.

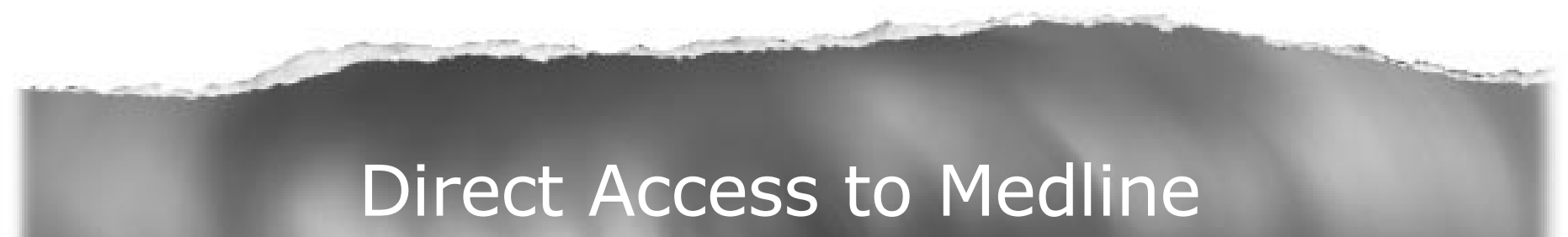

Medline

Link to Medline from the homepage and get straight into the National Library of Medicine's premier bibliographic database. Medline allows you to search across 9 million records of bibliographic citations and author abstracts from approximately 3,900 current biomedical journals.

www.jnnp.com 